\title{
Periodic paralysis with transient compartment-like syndrome
}

INSERM

\section{Source}

INSERM. (1999). Orphanet: an online rare disease and orphan drug data base. Periodic paralysis with transient compartment-like syndrome. ORPHA:397755

Periodic paralysis with transient compartment-like syndrome is a rare, genetic, neuromuscular disease characterized by normokalemic episodes of painful muscle cramping followed by progressive, permanent, flaccid weakness, triggered by stress, cold and exercise, associated with myopathic myopathy and painful acute edema with neuronal compression, foot drop and muscle degeneration when located in the tibialis anterior muscle group. 